\title{
ON THE RULES FOR THE CORRECTION OF ASTIGMATISM.
}

BY

J. W. NORDENSON,

LECTURERR ON OPHTHALMOLOGY AT THE ROYAL CAROLINE INSTITUTE, STOCKHOLM.

Although the question, whether in a given case the astigmatism of an eye should be corrected or not, occurs daily, and is important and complex, it has been little debated in ophthalmic literature, and the text-books give only vague directions on the subject. I venture, therefore, to review shortly the rules for and against the correction of astigmatism.

\section{Indications for the correction of astigmatism.}

In general, correction is needed only if it increases visual acuity. An exception to this rule will be mentioned later when the contraindications are being discussed. When correction improves acuity, it may beradvisable on account of the following :-

I. The need of accurate detail-vision.-This point is so obvious that it hardly needs discussion. It should be remembered, however, that, although a visual acuity considerably below normal is sufficient for most occupations, the education of the human mind is based largely on visual perceptions, and the more accurate these are the better. It is, therefore, often right to give correction, although the vision without it is sufficient for the patient's occupation. This applies, of course, more to children than to older persons.

II. Asthenopia - chiefly of two kinds :-

(a) Accommodation asthenopia.-In accommodating the pupil contracts; this cuts off the peripheral rays, and thereby tends to improve definition. But some ametropes, in order to obtain the maximum of this effect, accommodate in excess of what gives the proper focal distance. This spoils definition, and so counteracts the good effect of the contraction of the pupil. The patient, therefore, relaxes accommodation. The pupil thereby widening, he reverts to accommodation. If this alternation goes on for long, it produces fatigue and strain-accommodation asthenopia.

(b) Eyelid asthenopia. - The second kind of asthenopia is due to the strain of the orbicularis muscle. This muscle can be used to correct astigmatism, its palpebral portion exerting pressure on the globe. As this pressure cannot be exerted in any but a vertical direction, it is only inverse astigmatism that can be thus corrected. In this way as much as 2 or 3 dioptres can be compensated, but the continual straining of the orbicularis leads to fatigue, and may constitute an urgent need for cylindrical glasses.

Asthenopia arising from astigmatism has been considered a common cause of such nervous troubles as epilepsy, migraine, 
headache, etc., and it cannot be denied that there is a connection between these affections and astigmatism, and this is a good reason for correction in these cases.

III. Dangers to the eye involved by astigmatism.-Astigmatism may favour the development of strabismus or of myopia.

The development of strabismus is often due to the fact that the faculty of fusion is strained at the time when the desire for single vision is diminished. This diminishing of the desire for single vision occurs if one eye has less sharp retinal images than the other. The patient then suppresses the images of the defective eye, and takes no trouble to direct it so as to obtain single vision; he leaves it to take the position most convenient to its muscles.

Astigmatism in one eye, or astigmatism of different degrees in the two eyes, may, therefore, cause strabismus. It is, therefore, important that astigmatism should be corrected as soon as strabismus seems imminent. In certain cases the correction may at the same time help to prevent amblyopia ex anopsia. And if strabismus has already developed, the correction is likewise indicated as tending to promote fusion.

As to the relations between astigmatism and myopia, opinions depend ultimately on the views held as to the ætiology of myopia. It is generally admitted that close work plays a prominent part in the development of myopia. Astigmatism, by lowering visual acuity, induces the patient to bring the work close to the eyes, or the eyes down to the work; so, if myopia shows signs of developing, the astigmatism should be corrected. And in the case of inverse astigmatism, if the patient uses the orbicularis in the way described above, this, too, may promote myopia.

\section{Contra-indications to correction.}

I. The inconvenience of wearing glasses.-Only the case in which one eye is normal and the other astigmatic need be discussed here. In such cases it is generally not necessary to correct; sometimes, however, it is necessary, as, for instance, if the astigmatism is causing asthenopia, or if strabismus is threatening. Further, it might be of use to correct the single astigmatic eye if the patient needs very accurate binocular vision or a very wide field of vision, as might be the case with military men, for instance. If for some other reason the question of correction of the other eye occurs, astigmatism in the one eye further indicates correction.

II. Non-improvement of visual acuity by correction.-If an eye, though astigmatic, sees no better when corrected, it is generally useless to prescribe the cylinder unless it is required for some other purpose. And, if useless, glasses are an unnecessary expense and trouble. Sometimes, however, it may be advisable to correct even such an eye, namely, an eye in which correction has apparently no 
effect on acuity. One often meets with cases of astigmatism, particularly of direct astigmatism, in which correction gives at first no improvement. If, in these cases, correcting glasses be worn notwithstanding, one finds after a time, sometimes months, sometimes years, the patient comes back with considerably better acuity ; it may be increased, for instance, from $6 / 24$ to $6 / 8$ or better. This fact has been observed by many colleagues, but as far as I have seen, it has been very little discussed in the literature.

How is this increase of visual acuity to be accounted for? First, all sources of error must be excluded. There are mainly two such sources: (1) Very often at the first examination a patient unacquainted with the methods does not give exact answers, and does not read the test types, which he is quite well able to distinguish. It is well known to every practitioner, that especially children, when examined again on a subsequent day, often show decidedly better acuity. In the cases of this kind which I have seen, and from which I have attempted to draw any conclusion of this sort, the examination has, therefore, always been repeated at least once some days later. (2) The other source of error is variation in the size of the pupil. If the pupil is of different width on the two occasions on which the patient is examined, this will influence the degree of astigmatism found, and the acuity of vision. It must, therefore, be ascertained that the pupil is of the same diameter on both occasions. I have done this by Haab's method, which seems sufficiently accurate for this purpose.

These sources of error having been excluded, it seems justifiable to suppose that the acuity has really been increased. The explanation seems to be as follows:-When the eye is corrected the retinal image becomes sharper and the diffusion-circles smaller. Now a person who has been long accustomed to large diffusioncircles has probaby developed a faculty of interpreting them. When these diffusion-circles are suddenly reduced he will not at first be able to interpret fully the sharper retinal image. But after some time, when he is familiar with the new conditions, he will read more of the test-types.

The fact that correction of astigmatism does not immediately improve acuity is not, therefore, a final contra-indication to correction, which latter should be omitted only if the acuity remains unimproved after the use of glasses for some time. It seems advisable, therefore, especially in young children, to try correction for some time, and discontinue it if it proves useless.

III. Intolerance of correction.-In many cases the patient cannot tolerate correction, although this has been accurately determined and gives a considerable increase of acuity. This fact is often observed. Burnett* writes as follows: "It is a fact,

"Burnett, S. W. - "A Treatise on Astigmatism," p. 163. St. Louis, 1887. 
however, which occurs with unfortunate frequency in our clinical experience, that glasses which give perfect optical correction, particularly if it is determined under atropin, cannot be worn with comfort and sometimes not at all. Such cases are to be found as a rule in persons who have passed their youth with their anomaly uncorrected." Burnett endeavours to explain them. "I can only account for them," he says (p. 164), "on the supposition that the eyes have been accustomed for so long to the astigmatic state as to make the abnormal, in a certain sense, the normal condition, and that the cerebral centre for vision resents an interference with the established order of things. It must be remembered in this connection that in dealing with the human eye we have to do not with an optical instrument alone, but with an organ of sense as well. All of our senses are in a measure affected by education, and after a certain habit has once been firmly fixed it is with difficulty changed in any important particulars."

These opinions are illustrated by two cases out of his own practice, and probably all oculists could confirm them from their own experience. It occurs fairly often that a patient cannot tolerate the correction prescribed for his astigmatic eye, and the explanation given above seems to be a very likely one. It occurs mostly with patients of a certain age, and especially in those cases where the axes of the optical system of the eye do not coincide with the vertical and horizontal plane through the system, but run in a more or less diagonal direction. These patients are accustomed to distorted images in which right-angles appear acute or obtuse, and vertical and horizontal lines appear more or less diagonal ; but they know by experience the real shape of the objects thus misrepresented ; their cerebral centre of vision has learnt to interpret the images correctly. In such a case when the astigmatism is first corrected the correct retinal image is misinterpreted, so that a right-angle appears acute or obtuse.

But the mechanism of interpretation can be re-modelled to a certain extent, and the patient succeeds in a short time in interpreting correctly the new retinal image. But in some people this is only attained after considerable trouble. As is the case with all faculties depending on exercise, it is far easier for the young person than for an elderly one to re-educate his mechanism of interpretation. It is therefore only natural that the difficulties should increase with age.

The question is now whether such patients should be encouraged to try to accustom themselves to the correction or whether it should be discontinued. The age of the patient and the nature of his occupation will decide. Elderly people should generally not be pressed to continue to wear the correction in such cases, as they often seem to suffer a great deal from it. In young people one should persuade them to persevere, as it often happens that after 
some weeks, they have accustomed themselves to the glasses. In these cases it may often be advisable to correct the astigmatism by degrees, giving a weak cylinder first, letting the patient accustom himself to it, and gradually increasing it. In giving glasses for presbyopia the correction of a monocular astigmatism is generally not tolerated by the patient, and should be omitted. The same applies often to the correction of young hypermetropes for close work.

IV.- In a remarkable article Lagrange* pleads very urgently for not correcting astigmatism in children. He cites a number of cases in which astigmatism had been found and glasses prescribed but not worn, and in which, after a lapse of time, the patients returned with better vision and less corneal astigmatism than before. He is of opinion that this self-cure of astigmatism is due to the generally admitted tendency of the eye to become inversely astigmatic in old age, and as he has found no such change of astigmatism in eyes that had been properly corrected he considers correction an impediment to this natural alteration of the eye. The mechanism of this spontaneous cure of , astigmatism consists, according to him, in the unequal contraction of the ciliary muscle in different meridians, this muscle pulling most strongly in the horizontal meridian, and thus increasing the curvature of the cornea in this meridian. He, therefore, advises not to correct the hypermetropic astigmatism so often found in children unless it is causing definite trouble. If it is causing trouble, spherical glasses should be ordered first, so as to oblige the patient still to make efforts of astigmatic accommodation. If, however, the spherical correction should prove insufficient, one should proceed sparingly to cylindrical correction, and be satisfied as soon as the troubles of the patient are relieved, and his acuity of vision is sufficient for his work.

In the light of our modern conceptions of the mechanism of accommodation, this explanation of the self-cure of astigmatism seems untenable. The theory appears to be based on too few cases. For the present the possibility of self-cure by leaving the astigmatism uncorrected is not sufficient to contra-indicate correction in these cases.

The above indications and contra-indications do not exhaust the list ; moreover, they are, of course, not absolute, but must be weighed one against another according to the individual needs of every case.

"Lagrange.-Archives d'Ophtalmologie, 1909, p. 705. 